EESTI NSV TEADUSTE AKADEEMIA TOIMETISED. 31. KOIDË FOOSIKA * MATEMAATIKA. 1982, NR. 3

ИЗВЕСТИЯ АКАДЕМИИ НАУК ЭСТОНСКОП ССР. ТОМ 31 ФИЗИКА * МАТЕМАТИКА, 1982, № 3

\title{
ВЛИЯНИЕ КУМУЛЯЦИИ ИМПУЛЬСА АТОМА НА ЕГО СПЕКТР
}

\author{
(Представил К. К. Ребане)
}

В лазерной спектроскопии сейчас успешно решается задача полного управления движением единичных атомов [ $\left.{ }^{1}\right]$. Лазерный луч подходящего направления и подходящей частоты может управлять движением единичного атома или путем прямого светового давления, или через индуцированный дипольный момент атома при наличии градиента светового поля (см., напр., $\left.\left[{ }^{2}\right]\right)$. Такое управление приводит к эффектам фокусировки $\left[{ }^{3,4}\right]$, охлаждения $\left[{ }^{5-7}\right]$ и пленения $\left[{ }^{8,9}\right]$ атомов, а также ионов $\left[{ }^{10-12}\right]$. Оптическое детектирование единичных атомов, сотни раз переизлучивших фотоны в лазерном луче, проведено в $\left[{ }^{13}\right]$.

В связи с этим направлением лазерной спектроскопии представляет интерес исследовать особенности спектра единичного атома. В данной работе рассматривается кумуляция импульса на свободном единичном атоме в процессе многократного поглощения и излучения фотонов и проводится модельный расчет влияния кумуляции на форму спектра резонансной флуоресценции ансамбля единичных атомов.

Рассмотрим единичный свободный двухуровневый атом в резонансном электромагнитном поле лазерного луча. Обозначим основное и возбужденное состояния атома индексами 1 и 2 соответственно, а энергию перехода $1 \rightarrow 2$ в атоме - через $\hbar \omega_{21}$.

Рассмотрим многократное поглощение и излучение фотонов атомом. При поглощении фотона импульс атома увеличивается на импульс фотона $\vec{\hbar}_{\text {погл }}$, а при излучении фотона атомом импульс последнего увеличивается на импульс отдачи $-\vec{\hbar}_{\text {изл. }}$ При этом в спонтанном излучении обратные направления вектора $\vec{k}_{\text {нзл }}$ равновероятны, а в вынужденном излучении вектор $\vec{k}_{\text {изл }}$ параллелен волновому вектору вынуждающего излучения. Средний импульс отдачи от $N$-кратного спонтанного излучения равен нулю. Если спонтанное излучение сильно преобладает над вынужденным, то при условии $\vec{k}_{\text {погл }} \approx$ const на атоме кумулируется импульс $\approx N \vec{\hbar}_{\text {погл. }}$.

Кумуляция импульса на атоме смещает резонансную частоту поглощения и излучения из-за эффекта Допплера. Сохранение энергии и импульса при взаимодействии электромагнитного поля с атомом определяет резонансную частоту поглощения (๓пгл (с точностью до естественной ширины перехода $1 \rightarrow 2$ ) равенством

$$
\hbar \omega_{\text {погл }}=\hbar \omega_{21}+R+\vec{\hbar}_{k_{\text {погл }}}{\overrightarrow{v_{1}}}_{1} \text {, }
$$

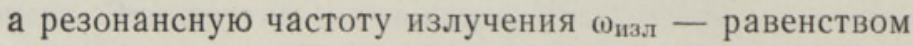

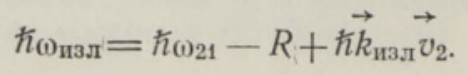


Здесь $R=(\hbar \omega)^{2} / 2 M c^{2}-$ энергия отдачи, $M-$ масса атома, $\overrightarrow{v_{1}}$ и $\overrightarrow{v_{2}}-$ скорости атома в состояниях 1 и 2 соответственно.

Прирост импульса на величину $\approx N \pi \vec{k}_{\text {погл }}$ (знак $\approx$ означает, что $\hbar \vec{k}_{\text {погл }}$ здесь принят без учета зависимости резонансной частоты поглощения от скорости) приводит к изменению скорости атома на

$$
\Delta \vec{v}=N \div \vec{k}_{\text {погл }} / M
$$

По (2) это дает допплеровское смещение резонансной частоты излучения, измеренного при условии $\vec{k}_{\text {нгт. }} \| \vec{k}_{\text {погл, }}$, на величину

$$
2 N R \text {. }
$$

Для атома водорода энергия отдачи при $\hbar_{\omega}=1$ эB составляет $R=4,3 \cdot 10^{-6} \mathrm{~cm}^{-1}$. В $\left[{ }^{13}\right]$ был поставлен эксперимент с многократным поглощением и излучением фотонов единичным атомом $\mathrm{Na}$ в резонансном лазерном луче, где $N$ оценено равным 250. Для такого атома энергия отдачи $\left(\pi_{\omega} \approx 2{ } B\right)$ равна $7 \cdot 10^{-7} \mathrm{CM}^{-1}$, а смещение (4) $4 \cdot 10^{-4} \mathrm{~cm}^{-1}$. Скорость (3) изменяется при этом на $7 \mathrm{Mc}^{-1}$.

Рассмотрим далее кумуляцию импульса как вероятностный процесс, описываемый в ансамбле единичных атомов следующей системой кинетических уравнений *

$$
\begin{gathered}
\dot{p}_{1}(v, t)=\beta(v) p_{2}(v+1, t)-\beta(v) p_{1}(v, t)+\alpha p_{2}(v, t), \\
\dot{p}_{2}(v, t)=\beta(v-1) p_{1}(v-1, t)-\beta(v-1) p_{2}(v, t)-\alpha p_{2}(v, t) .
\end{gathered}
$$

Здесь $p_{1}(v, t)$ - число атомов в оснозном состоянии, обладающих скоростью $v$ в момент времени $t, p_{2}(v, t)$ - число атомов в возбужденном состоянии, $v-$ проекция скорости атома на волновой вектор $\vec{k}_{\text {погл }}$, $\beta(v)$ - скорость поглощения или вынужденного излучения, $\alpha-$ скорость спонтанного излучения. За единицу скорости $v$ выбрана $九 k / M$. $\beta(v)$ пропорциональна интенсивности лазерного луча при резонансной для скорости $v$ частоте $\omega_{\text {погл }}$ (с точностью до естественной ширины перехода $1 \rightarrow 2$ ).

Скорость атома увеличивается на $\hbar k / M$ при поглощении и уменьшается на $\bar{\hbar} k / M$ при вынужденном излучении. Изменение скорости при спонтанном излучении не учитывается, так как средняя скорость при многократном спонтанном излучении не меняется. Кумуляция импульса на атоме кончается, если атом выходит из лазерного луча или кумуляция выводит его из резонанса.

В описываемый уравнениями (5) ансамбль следует включить атомы с резонансными скоростями $v$, обеспечивающими по формуле (1) резонанс атомного перехода $1 \rightarrow 2$ с поглощаемыми частотами лазерного луча. Система уравнений (5) связывает резонансные скорости, отличающиеся на $\hbar k / M$. Поэтому в интервале непрерывных значений резонансных скоростей будем учитывать только дискретные значения интервалами $\hbar k / M$.

Если провести счет фотоноз спонтанного излучения частоты $\omega_{\text {изл }}$ с волновым вектором $\vec{k}_{\text {нзл}}$, практически параллельным вектору $\vec{k}_{\text {пог.л }}$, результат будет для промежутка времени $(0, T)$ пропорционален величине

$$
\Phi\left(\omega_{\text {пзлл }}\right)=\Phi(v)=\int_{0}^{T} d t p_{2}(v, t) .
$$

* Такой вероятностный подход означает отказ от когерентных эффектов и оправдан в предположении сильного преобладания спонтанного излучения над вынужденным. 


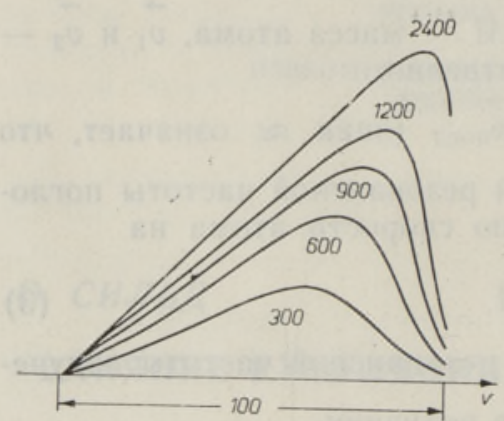

Рис. 1. Интегральный спектр резонансной флуоресценции ансамбля атомов в зависимости от времени $T$ их взаимодействия с лазерным лучом.

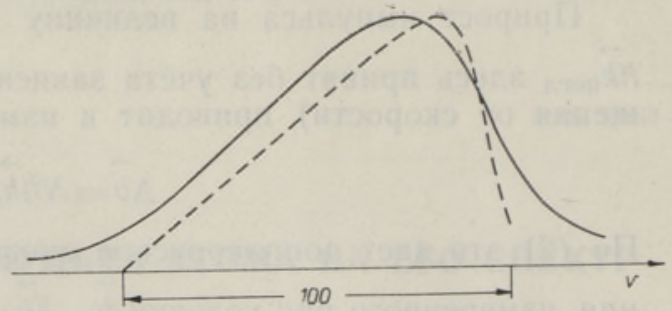

Рис. 2. Интегральный спектр резонансной флуоресценции в случае, когда естественная ширина атомной спектральной линии сравнима с частотной шириной лазерного луча. Пунктирная кривая - распределение интенсивностей естественных контуров.

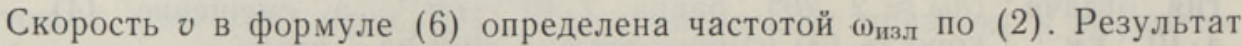
счета фотонов пропорционален (6) в предположении, что естественная ширина атомной спектральной линии существенно меньше ширины лазерного луча. Если это не так, формула (6) определяет распределение интенсивностей естественных контуров.

Предполагая все резонансные скорости в ансамбле равновероятными в начальный момент, систему уравнений (5) следует решать при начальных условиях: $p_{2}(v, 0)=0, p_{1}(v, 0)=$ const.

Можно проинтегрировать кинетические уравнения (5) и установить следующую рекуррентную формулу для спектра (6) :

$$
\Phi(v+1)=\Phi(v)+1-p_{1}(v, T)-p_{2}(v+1, T)
$$

(предположена нормировка $\alpha=1$ и $p_{1}(v, 0)=1$ ).

При малых $T$ спектр (6) близок к частотному спектру распределения интенсивностей в лазерном луче, так как сдвиг резонансных частот под влиянием кумуляции еще мал. В прсделе $T \rightarrow \infty$ кумуляция выводит из резонанса все атомы, т. е. $p_{1}(v, \infty)=p_{2}(v, \infty)=0$, и спектр (6) становится линейным по шкале скоростей $v$ (см. формулу (7)).

На рис. 1 показан спектр (6), рассчитанный для промежутка резонансных скоростей $\Delta v=100$ с гауссовым распределением интенсивностей в лазерном луче (с полушириной $\delta v=48$ ). При максимальной интенсивности луча отношение $\beta / \alpha$ принято равным 0,1 . Как видно, форма спектра, при малых промежутках измерения $(0, T)$ близкая к гауссовой, при возрастании $T$ приближается к линейной. Здесь спектр рассчитан без учета естественной ширины атомной спектральной линии. За единицу времени принято оптическое время жизни атома.

Рис. 2 (пугктирная кривая - функция $\Phi(v)$ по (6)) показывает спектр с учетом естественной ширины (по шкале скоростей ей соответствует полуширина распределения $\delta v=24)$.

Таким образом, лазерный луч взаимодействует с ансамблем свободных атомов с резонансными скоростями. Ширина и форма спектра резонансной флуоресценции ансамбля сначала определяются частотной шириной и формой лазерного луча (с точностью до естественной ширины резонансного перехода атома), если все резонансные скорости ансамбля атомов равновероятны.

В результате достаточно длительного взаимодействия лазерного луча с ансамблем кумуляция импульса ускоряет все атомы до экстремальной резонансной скорости. После этого в спектре флуоресценции остается только соответствующая экстремальная резонансная частота, 
а остальные выжигаются. Именно для такого процесса в ансамбле свободных атомов рассчитан выше интегральный переходный спектр флуоресценции, асимметричный и смещенный, а без учета естественной ширины линейно возрастающий в сторону экстремальной резонансной частоты. Нужное для этого процесса число переизлучений $N$ следует оценить величиной $\hbar \Delta \omega / 2 R$, если $\llcorner\omega$ - ширина интервала резонансных частот.

Автор благодарен К. К. Ребане за предложение темы работы и ее обсуждение.

\section{Л И Т Е Р А Т У Р А}

1. Летохов В. С., в кн: Примененне лазеров в атомной, молекулярной и ядерной физике (Труды I Всесоюзной школы, Вильнюс, 21-31 августа 1978 г.), М., «Наука», 1979, с. $24-40$.

?. C.ook, R. J., Phys. Rev. A, 20, № 1, 224-228 (1979).

3. А ск ар вян Г. А., Ж. эксперим. и теор. физ., 42, вып. 6. 1567-1570 (1962).

4. B jorkholm, J. E., Freeman, R. R., Ashkin, A., Pearson, D. B., Phys. Rev. Lett., 41, № 20, 1361-1364 (1978).

5. Hänsch, T. W., Schawlow, A. L., Opt. Commun., 13, № 1, 68-69 (1975).

6. Л етохов В. С., Миногин В.Г., Пав лик Б. Д., Ж. эксперим. и теор. физ., 72, вып. 4, 1328-1341 (1977).

7. W ineland. D. J., It a no, W. M... Phys. Rev. A. 20, № 4, 1521-1540 (1979).

8. Л е тохов В. С., Письма в ЖЭТФ. 7, вып. 9, 348-351 (1968).

9. Ash k i n, A., Phys. Rev. Lett., 40, № 12, 729-732 (1978).

10. Wineland, D. J., Drull inger, R. E., Wa11s, F. L., Phys. Rev. Lett., 40. $1639-1642(1978)$.

11. Neuhauser. W., Hohenst at t, M., Toschek, P., Dehmelt, H., Phys. Rev. Lett., 41, 233-236 (1978).

12. Javan a inen, J., S te nholm, S., Appl. Phys., 21, 35-45 (1980); 21, 163-167 $(1980): 21,283-291(1980)$

13. Ба лыкин В. И., Беков Г. И., Л етохов В. С., Мишин В. И., в кн.: Применение лазеров в атомной, молекулярной и ядерной физике (Труды I Всесоюзной школы, Вильнюс, 21-31 августа 1978 г.), М., «Наука», 1979, с. 41-59.

Ннститут физики
Академии наук Эстонской ССР

Поступила в редакцию

$11 / \mathrm{I} 1982$

O. SILD

IMPULSI KUMULATSIOONI MOJU AATOMI SPEKTRILE

Oksikul vabal aatomil kumuleerub footonite korduva neeldumise ia spontaanse kiirgamise protsessis impulss, mis nihutab laserikiire ja aatomi resonantsi sagedust Doppleri efekti tōttu. Töös on arvutatud impulsi kumulatsiooni mõiu vabade aatomite ansambli fluorestsentsispektrile. Ansambli piisavalt kestev interaktsioon laserikiirega kiirendab aatomeid seni, kuni nad saavutavad resonantsi piirsageduse. Sellega kaasneb teiste sageduste väljapõletamine fluorestsentsispektris.

O. SILD

\section{EFFECT OF MOMENTUM CUMULATION ON ATOMIC SPECTRUM}

The momentum cumulation in the process of repetitive photon absorption and spontaneous emission in a free single atom is considered. The momentum cumulation effect on the resonance fluorescence spectrum of the ensemble of two-level atoms is calculated.

The resonance frequencies of absorption/emission are determined by the formulae (1)/(2), depending on the velocities $\overrightarrow{v_{1}} / \vec{v}_{2}$ of the atoms in the ground/excited states. The momentum cumulation shifts the resonance frequency due to the Doppler effect. If the interaction between the atoms and laser field is sufficiently long-lasting, the momentum cumulation accelerates all the atoms up to the extreme resonance velocity. As a result, in fluorescence spectrum only the corresponding extreme resonance frequency is conserved, the others being burned out.

In this paper, for the preceding process the integrated transient spectrum of resonance fluorescence is calculated (see Fig. 1, where $T$ is the time interval of the interaction between the atoms and laser field). With $T$ increasing, the spectrum shifts towards the extreme resonance frequency and becomes asýmmețical. 